\title{
The Role of Features in Chinese Character Recognition
}

\author{
Feifan Luo, Ye Zhang \\ Institutes of Psychological Sciences, Hangzhou Normal University, Hangzhou, China \\ Email: luofeifan0311@sina.com
}

How to cite this paper: Luo, F.F. and Zhang, Y. (2020) The Role of Features in Chinese Character Recognition. Open Access Library Journal, 7: e6358.

https://doi.org/10.4236/oalib.1106358

Received: April 25, 2020

Accepted: May 16, 2020

Published: May 19, 2020

Copyright (c) 2020 by author(s) and Open Access Library Inc.

This work is licensed under the Creative Commons Attribution International License (CC BY 4.0).

http://creativecommons.org/licenses/by/4.0/

\begin{abstract}
Chinese characters contain three levels of visual information: strokes, radicals, and structures. Stroke, as the basic unit of Chinese characters, is the foundation of the formation of Chinese characters. Some research has shown that strokes can enhance the semantic and orthographic representation in the process of Chinese character acquisition. This shows that the processing of Chinese characters is based on feature processing. This is consistent with the feature processing theory of letter and object recognition. Recent research on visual recognition shows that the recognition of letters and words is based on the termination and the other features (vertices and midsegment) used in object recognition. In this study, we used delayed segment technique on Chinese character recognition to examine the importance of terminations, vertices and midsegment in different masking degree. Experiment 1 selected about 43 high frequency Chinese characters with left-right structures and asked the participants to naming Chinese characters. In experiment 1 , we try to examine the importance of terminations, vertices and midsegments based on line unit and consider the degree of masking these features. It was found that the main effect of masking degree was significant. The masking of $35 \%$ and $55 \%$ of the features makes the RTs significantly longer. The main effect of feature type was significant. Masking vertices and midsegment can make RTs significantly longer. But termination didn't show its feature effect in experiment 1 . When masking these three features (vertices, midsegment and termination) in experiment 1 , the features are based on line units, which may destroy the integrity of strokes in Chinese characters. That termination effects couldn't be obvious in experiment 1 may be caused by this reason. To verify this, in experiment 2, we explore the vertices, midsegment and termination based on stroke units. The results show that the main effect of masking degree was significant, and the RTs were longer by masking $35 \%$ and $55 \%$ degree. The main effect of feature types was significant. RTs on character recognition are significantly longer by masking the vertices, midsegment and
\end{abstract}


termination. This shows that the vertices, midsegment and termination based on stroke unit are also important in Chinese character recognition. Based on the above research results, it can be seen that the key features on Chinese character recognition are in line with the neuronal recycling hypothesis. According to neuronal recycling hypothesis, human's ability to learn from culture depends on the retrieval of preexisting brain circuits, and the key features in word recognition may be derived from our key features in object processing. The hook is considered to be a special termination in Chinese characters. The results of experiment 2 also emphasized the key role of hook features in character recognition.

\section{Subject Areas}

Psychology

\section{Keywords}

Word Recognition, Chinese Stroke, Visual Features, Masking

\section{Introduction}

The first step of Chinese character recognition is the procession of the character shape, so the problem of the basic processing unit has become the focus of linguists and psychologists. What are the functions of strokes, radicals, shape in the recognition of Chinese characters? There is still considerable disagreement among psychologists. Some studies focused on whole character; they believed that the cognition of Chinese characters is in essence a holistic process, and the whole character is the basic unit of Chinese character recognition. Other studies focused on the role of features in Chinese character recognition. They believe that Chinese character is processed from features to whole character, and features play an important role on this process. Strokes and radicals are the basic units of Chinese character recognition.

\subsection{The Role of the Stroke}

Stroke is a continuous movement of a pen on the paper; while it is not the same stroke any more when the pen leaves the paper and then touches the paper to retrace the movement. It is interesting to note that strokes as being the smallest units in Chinese character have intrinsic differences with radicals. For instance, radicals could convey semantic or phonological information of whole characters. For example, the character 伦 ([lun], "ethical”) has two radicals at the left-right position, where the right radical $仑$ is a phonetic radical providing phonological cue and the left radical $\uparrow$ is semantic radical indicating semantic category. In contrast, strokes don't have these functions. Positions of strokes in characters are flexible. Different from radicals arranged usually horizontally and vertically, strokes could be placed on anywhere at the characters plane. Single character is 
only made up of strokes and can't be divided into components (for example, 与 [yu] "and").

Since each Chinese character is a combination of a number of strokes that occupies an area spatially separated from characters, some factors within the characters have also been found to affect the recognition of Chinese characters. Flores B d'Arcais [1] investigated the effects of "early" and "late" strokes (depending writing orders of a character) on whole character identification. They found that "early" strokes can carry more information of a Chinese character than the "late" strokes. Yan et al. [2] removed several strokes in characters to exam the role of stroke order coding in Chinese character identification during reading. By using eye movements technique, researchers have proposed not all strokes contain equal importance to character identification. Tseng et al. [3] used stroke removing task in sentence to estimate the redundancy of Chinese strokes and the relative information in which the subjects were asked to fill stroke in characters. They find that the redundancy in Chinese (stroke as calculating unit) is higher than that in English (letter as calculating unit).

Zhang et al. [4] suggested that the identification of strokes has been shown to be unaffected by their complexity. One of the reasons is that the stroke may become a memory chunk. Perceptual systems tend not to subdivide it at the automatic processing stage. However, once a controlled process is applied, such as rating the complexity of the strokes, the differences in the complexity of the strokes are immediately apparent. This may be why stroke assessment has a complexity effect but stroke recognition does not.

\subsection{The Mechanism of Feature Processing Mechanism}

Selfridge put forward the "Pandemonium model" in 1959 [5]. In the way of feature analysis, Pandemonium model divides the recognition process into four levels, each level has some "Demon" to perform a specific task, these "Demon" work in a hierarchical order. The first level is the image of stimuli encoded by "Image demon". Then the "Feature demon" of the second level analyzes the image of the stimulus and decomposes it into various features. The function of "Feature demon" is unique, they only search the feature for their own, and reporting clearly on whether the stimulus has the corresponding feature and its number. In the third level, "Cognitive demon" always monitoring the reaction of the various "Feature demon", each "Cognitive demon" is responsible for their pattern; they will find the feature that is relevant of the pattern from the reaction of "Feature demon". When found relevant features, "Cognitive demon" will cry, the more features, the louder they cry. Finally, the "Decision demon" selects the pattern with the loudest cry as the pattern to be recognized. For example, when we recognize the letter $\mathrm{R}$, the image of $\mathrm{R}$ is first formed by the "Image demon", and the "Feature demon" search the relevant features: a vertical line, two horizontal lines, a slant right line, a left opened curve, and three right angles. Then "Cognitive demon" began looking for pattern including these features, and P, D 
and $\mathrm{R}$ will cry, but only $\mathrm{R}$ demon cry is the loudest because all features of $\mathrm{R}$ demon are same with relevant features, while $\mathrm{P}$ and $\mathrm{D}$ the demon is not completely consistent with the relevant features, so the "Decision demon" choose R pattern.

This model proves that letter recognition is realized through the hierarchical processing of features. Recent studies have provided evidence to support this feature processing model [6] [7] [8]. Fiset et al. obtained classification images from 26 lowercase and uppercase English letters, and found that the termination was the most important diagnostic information in letter recognition [6] [9]. Based on these findings, the latest research further examines the relative importance of features such as midsegment, vertices and terminations in word recognition [8]. They found that masking the midsegment significantly increased the difficulty of word recognition, which indicates that the midsegment plays an important role in visual word recognition.

In the present experiments, we examined the relative importance of midsegments, vertices, and terminals of Chinese character and explore how strokes unit influences these features. We select midsegments, vertices and terminations because they are the most frequently cited as important features of the letters in previous literature. Terminals were included because of the importance inferred for them from the Bubbles experiments. Unlike the features identified in the Chang et al. [10] work, vertices, midsegments and terminals are feature-bearing components of all letters. In our study, we conducted two lexical decision experiments. In Experiment 1, we compared different types of previews (vertices, midsegments and terminations) in different masking degree (masking $35 \%$ and masking 55\%). The way of masking features was based on line unit. For instance, midsegments were defined as the middle part of the line. Experiment 2 was prompted by the lack of Chinese strokes influence in Experiment 1. In Experiment 2 , in order to exam the effect of strokes, the way of masking features was based on stroke unit. In these experiments by using delayed segment technique we ask whether the vertices, midsegments and terminations play an important role in the recognition of Chinese character and if strokes unit could influence this feature effect.

\section{Experiment 1}

\subsection{Method}

\subsubsection{Participants}

Eighteen undergraduate and graduate students ( 9 males, $23 \pm 1.6$ years ago) from the Hangzhou Normal University took part in the study. All participants were native Chinese speakers and had normal or corrected-to-normal vision.

\subsubsection{Stimuli}

In experiment 1 , we used delayed segment technique to name Chinese characters. The delayed segment technique is similar to the delayed letter technique used by Lee et al. [11], with the only difference being that they preview the whole letter, whereas delayed segment technique preview partial Chinese character. In 
this technique, the preview stimuli is generated by partially masking the target stimuli, and the partially masked preview stimuli is immediately replaced by the target stimuli after $50 \mathrm{~ms}$, so as to show the importance of the masking part in this task [12] [13] [14]. In delayed segment technique, the baseline is included, and the unmasked target is also used as a preview to establish the baseline data.

In experiment 1, we selected 43 Chinese characters from 100 commonly used high frequency Chinese characters with left-right structure to explore vertices and midsegment effects, and 20 Chinese characters to explore terminations effects. The size of stimuli is $256 \times 256$ pixels; the mean width of Chinese character is 190 pixels. The mean word-frequency is 599 occurrences per million (range: 117.45 - 892.78). We used three ways of masking; vertices masking, termination masking and midsegment masking. In three different masking ways, we define the vertices as the intersection of two or more lines, define the midsegment as the middle part of the line, and define the termination as the endpoint of the line. At the same time, the masking degree of original character is also different, which is respectively masking $35 \%$ and $55 \%$ of the original character. So seven previews were created from each target: 1 ) the target itself (i.e. the whole preview condition); 2) a partial preview masking termination in 35\% degree; 3 ) a partial preview masking termination in 55\% degree; 4) a partial preview masking vertices in $35 \%$ degree; 5) a partial preview masking vertices in 55\% degree; 6) a partial preview masking midsegment in 35\% degree; and 7) a partial preview masking midsegment in 55\% degree; the previews are shown in Figure 1.

\subsubsection{Procedure}

The program runs on a Dell OptiPlex computer. The display is a 21-inch display with a refresh rate of $75 \mathrm{~Hz}$. The experimental program was written using PsychoPy3.0.

Before the experiment began, participants completed a set of 80 practice trials. Each trial started with the presentation of a fixation signal (+) for $500 \mathrm{~ms}$. Then, the preview was presented for $50 \mathrm{~ms}$ and was immediately followed by the target. The 50 ms previews could be one of seven types of preview. RTs were measured from the onset of the target until the participant's response (see Figure 2). All stimuli were presented in the same spatial location. The target remained on the screen until the participant made a response or $2 \mathrm{~s}$ had passed. Each subject completed 700 trials in the formal experiment, and 100 trials in each of the seven different types of preview.

\section{Results and Discussion}

Could the vertices, terminations and midsegment play a key role in Chinese character recognition? In order to solve this problem, in experiment 1, we selected high-frequency character with different left-right structures and masking their vertices, terminations and midsegment features to different degrees. The correct response time and accuracy of each preview in experiment 2 are shown in Table 1. 
Table 1. Mean lexical decision times (in ms) and accuracy (in parentheses) for chinese character in experiment 1.

\begin{tabular}{|c|c|c|c|c|c|c|c|c|}
\hline \multicolumn{9}{|c|}{ Experiment 1} \\
\hline Masking degree & \multicolumn{2}{|c|}{$35 \%$} & \multicolumn{2}{|c|}{$55 \%$} & \multirow{2}{*}{$\frac{0}{\text { Target }}$} & \multirow{2}{*}{$\begin{array}{c}35 \% \\
\text { Termination }\end{array}$} & \multirow{2}{*}{$\begin{array}{c}55 \% \\
\text { Termination }\end{array}$} & \multirow{2}{*}{$\begin{array}{c}0 \\
\text { Target }\end{array}$} \\
\hline Feature & Midsegment & Vertices & Midsegment & Vertices & & & & \\
\hline RTs (ms) & 426 & 422 & 437 & 428 & 411 & 414 & 421 & 420 \\
\hline Accuracy & $99 \%$ & $99 \%$ & $98 \%$ & $95 \%$ & $97 \%$ & $97 \%$ & $98 \%$ & $97 \%$ \\
\hline
\end{tabular}

In experiment 1 , as for Midsegment and Vertices, we found a main effect of preview type $\left(\mathrm{F}(2,84)=22.045, p<0.01, \omega^{2}=0.24\right)$ and masking degree $(\mathrm{F}(2,84)$ $\left.=24.991, p<0.01, \omega^{2}=0.27\right)$. For terminations, main effect of preview type $(t(19)=-0.555, p=0.586)$ and masking degree $(\mathrm{F}(2,38)=1.206, p=0.311)$ were not significant.

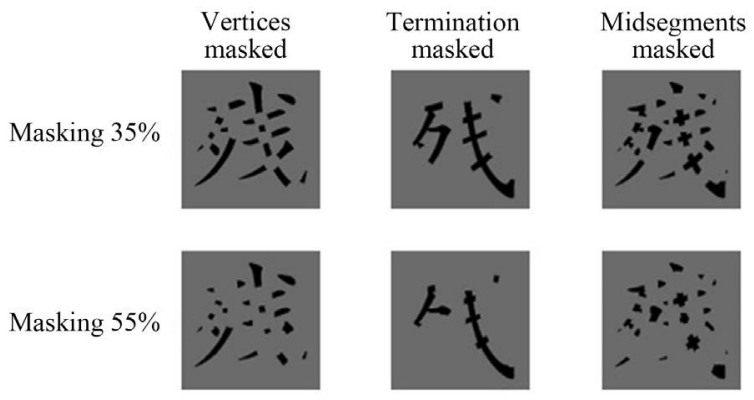

Figure 1. Previews were created from each target in experiment 1.

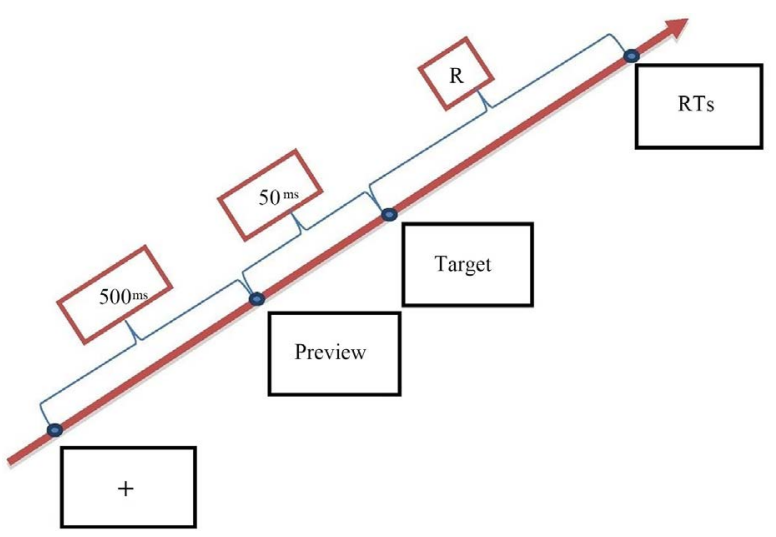

Figure 2. Delayed segment technique procedure in experiment 1 .

We firstly want to verify the vertices and midsegment effects, and we performed one-way repeated measurement ANOVAs for the features type and masking degree. As for the feature type (vertices, midsegment, baseline), we found that the feature effect was significant $\left(\mathrm{F}(2,84)=22.045, p<0.01, \omega^{2}=0.24\right)$. In the Post Hoc, the RTs between the midsegment $(431 \mathrm{~ms})$, the vertices $(425 \mathrm{~ms})$ and the target $(411 \mathrm{~ms})$ was significant, and the RTs between the vertices (424 $\mathrm{ms})$ and the midsegment (432 ms) was not significant ( $p>0.016(0.5 / 3)$, Bonferroni) (Figure 3).

Meanwhile, we found that the main effect of masking degree was significant $\left(\mathrm{F}(2,84)=24.991, p<0.01, \omega^{2}=0.27\right)$. In the Post Hoc, the 35\% masking degree (424 ms), 55\% masking degree (432 ms), and the baseline (411 ms) were significantly different in pairs $(p<0.016(0.5 / 3)$, Bonferroni) (Figure 4). 


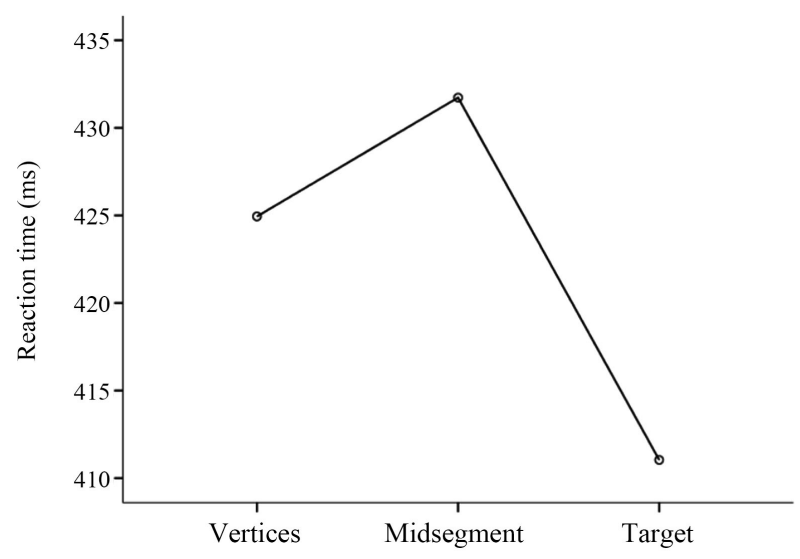

Figure 3. Effect of feature type on chinese character naming in experiment 1 .



Figure 4. Effect of masking degree on chinese character naming in experiment 1 .

Finally, we verified the termination effect. It was found that the feature main effect was not significant $(t(19)=-0.555, p=0.586)$. Subsequently, one-way repeated measurement ANOVAs was performed on the masking degree of the termination, and it was found that the main effect of the masking degree was not significant $(\mathrm{F}(2,38)=1.206, p=0.311)$.

The results of experiment 1 show that the midsegment and vertices play an important role in the recognition of Chinese characters, and the masking degree of $55 \%$ can effectively affect the correct recognition of Chinese characters. In experiment 1 , the three features (midsegments, vertices and terminations) definition, our principles are based on line unit to masking different features. In experiment 2 , based on the stroke unit to define the midsegments, vertices and terminations, we try to test whether the stroke unit could influence these feature effect.

\section{Experiment 2}

\subsection{Method}

\subsubsection{Participants}

Eighteen undergraduate and graduate students ( 9 males, $21 \pm 1.8$ years ago) 
from the Hangzhou Normal University took part in the study. All participants were native Chinese speakers and had normal or corrected-to-normal vision.

\subsubsection{Stimuli}

In experiment 2, we selected 44 Chinese characters from 100 commonly used high frequency Chinese characters with left-right structure to explore vertices and midsegment effects, and 20 Chinese characters to explore terminations effects. We also used three ways of masking; vertices masking, termination masking and midsegment masking and set up two making degree ( $35 \%$ vs $55 \%)$. The difference is that we have different definitions of the three features in experiment 2 . We take strokes as the basic unit to masking. We define the vertices as the intersection of strokes, define the midsegment as the middle part of strokes, and define the termination as the endpoint of strokes. The previews are shown in Figure 5.

\subsubsection{Procedure}

It was the same as in experiment 1 . Each subject completed 700 trials in the formal experiment, and 100 trials in each of the seven different types of preview.

\section{Results and Discussion}

In experiment 2 , we want to verify whether the vertices, terminations and midsegment defined based on the stroke unit still have the same results as in experiment 1 . The correct reaction time and accuracy of each preview in experiment 2 are shown in Table 2 .

We firstly want to verify the vertices and midsegment effects, and we performed one-way repeated measurement ANOVAs for the features type and masking degree. As for the feature type (vertices, midsegment, baseline), we found that the feature effect was significant $\left(\mathrm{F}(2,86)=26.653, p<0.01, \omega^{2}=0.28\right)$. In the Post Hoc, the RTs between the midsegment $(434 \mathrm{~ms})$, the vertices $(433 \mathrm{~ms})$ and the target $(411 \mathrm{~ms})$ were significant, and the RTs between the vertices (433 $\mathrm{ms}$ ) and the midsegment (434 ms) were not significant ( $p>0.016(0.5 / 3)$, Bonferroni) (Figure 6).

Meanwhile, we found that the main effect of masking degree was significant $\left(\mathrm{F}(2,86)=28.939, p<0.01, \omega^{2}=0.30\right)$. In the Post Hoc, the 35\% masking degree (429 ms), 55\% masking degree (438 ms), and the baseline (411 ms) were significantly different in pairs $(p<0.016(0.5 / 3)$, Bonferroni) (Figure 7).

Table 2. Mean lexical decision times (in ms) and accuracy (in parentheses) for chinese character in experiment 2.

\begin{tabular}{cccccccccc}
\hline \multicolumn{7}{c}{ Experiment 2} \\
Masking degree & \multicolumn{2}{c}{$35 \%$} & & $55 \%$ & 0 & $35 \%$ & $55 \%$ & 0 \\
\hline Feature & Midsegment & Vertices & Midsegment & Vertices & Target & Termination & Termination & Target \\
\hline RTs (ms) & 427 & 431 & 439 & 428 & 411 & 430 & 436 & 417 \\
Accuracy & $99 \%$ & $99 \%$ & $99 \%$ & $97 \%$ & $97 \%$ & $97 \%$ & $98 \%$ & $97 \%$ \\
\hline
\end{tabular}

In experiment 2, as for Midsegment and Vertices, we found a main effect of preview type $\left(\mathrm{F}(2,86)=26.653, p<0.01, \omega^{2}=0.28\right)$ and masking degree $(\mathrm{F}(2,86)$ $\left.=28.939, p<0.01, \omega^{2}=0.30\right)$. For terminations, main effect of preview type $(t(19)=3.037, p<0.05)$ and masking degree $\left(\mathrm{F}(2,38)=7.966, p<0.05, \omega^{2}=\right.$ $0.20)$ were significant. 


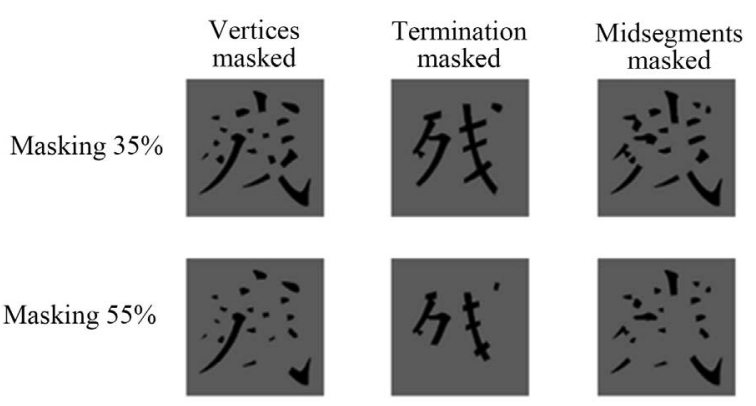

Figure 5. Previews were created from each target in experiment 2.

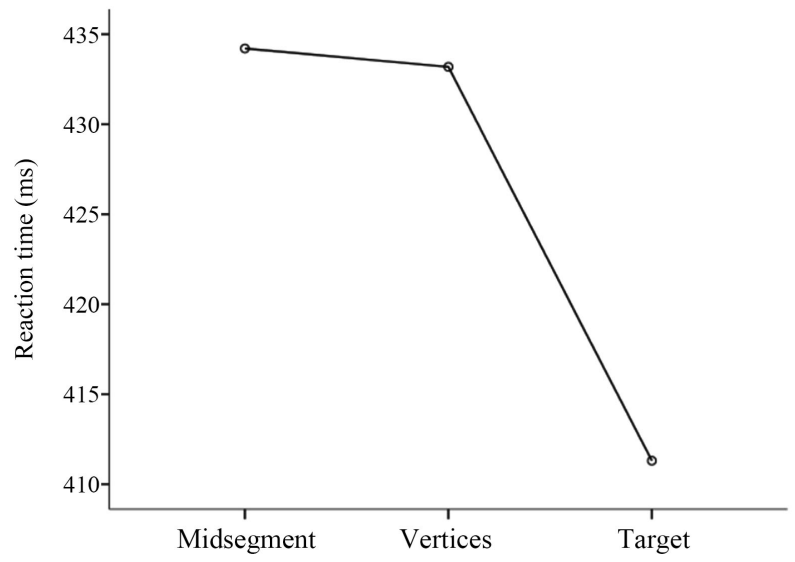

Figure 6. Effect of feature type on Chinese character naming in experiment 2 .

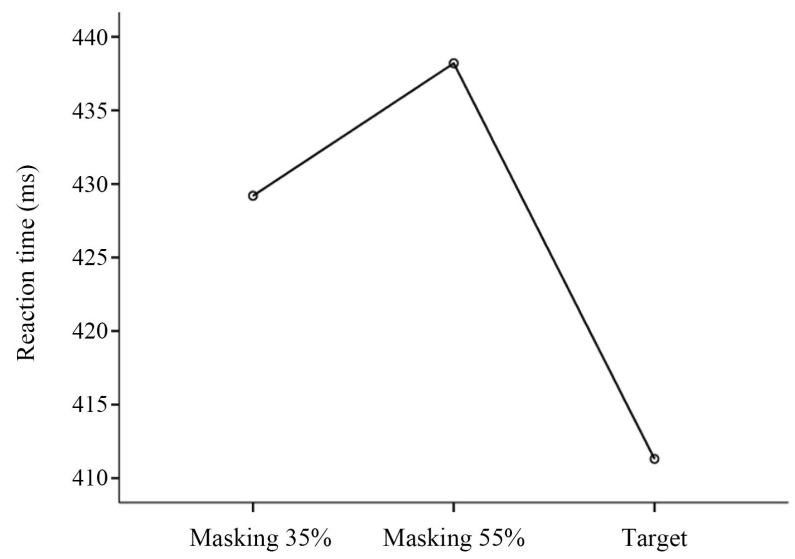

Figure 7. Effect of masking degree on chinese character naming in experiment 2 .

Finally, we verified the termination effect. It was found that the feature main effect was significant $(t(19)=3.037, p<0.05)$. Subsequently, one-way repeated measurement ANOVAs was performed on the masking degree of the termination, and it was found that the main effect of the masking degree was significant $\left(\mathrm{F}(2,38)=7.966, p<0.05, \omega^{2}=0.20\right)$. In the Post Hoc, it was found that the difference between masking $55 \%$ (437 ms) and baseline was significant, while the difference between masking $35 \%$ (430 ms) and baseline (417 ms) was not signif- 
icant $(p>0.16(0.05 / 3)$, Bonferroni).

The results of experiment 2 show that the vertices, terminations and midsegment defined based on stroke unit indicate their importance in Chinese character recognition. The degree of masking was significantly important at the vertices, terminations and midsegment. This shows that the features such as vertices, terminations and midsegment based on the definition of stroke unit also play an important role in character recognition.

\section{General Discussion}

The purpose of the experiment 1 and experiment 2 is to verify which features are critical in naming Chinese characters. For this reason, we used the delay segment technique to set up different previews, which would be presented for $50 \mathrm{~ms}$ before the presentation of the target. We verified whether the masking preview had an impact on Chinese character recognition by recording the responses of the participants.

In experiment 1 , we mainly verify the influence of vertices, terminations and midsegment on Chinese character recognition. As the introduction shown below, vertices, terminations and midsegment play an important role in English word recognition. In addition, we also added the independent variables of masking degree, the levels of which were $35 \%$ and $55 \%$ (relative to the target). Through the treatment of the masking degree, we hope that character recognition can be influenced by vertices, terminations and midsegment from enough masking degree. In experiment 1, the preview is the vertices, terminations and midsegment features of different masking degrees. The experimental task is using the delayed segment technique to name the left-right structural Chinese characters. The results of experiment 1 show that the feature effect is significant, and the midsegment and vertices play a key role in Chinese character recognition. Compared with the baseline response, the effects of midsegment and vertices are $21 \mathrm{~ms}$ and $14 \mathrm{~ms}$ respectively. However, the effect of the termination was not significant, and there was no significant difference from the baseline response. At the same time, the masking degree effect in experiment 1 is significant, and the masking degree of $55 \%$ and $35 \%$ will significantly increase RTs.

In experiment 2, we mainly want to verify the influence of the vertices; terminations and midsegment based on the definition of stroke unit on Chinese character recognition. Stroke is the most basic unit of Chinese characters. We want to know whether there have structural features based on the relationship between strokes that affect the cognition of Chinese characters. In experiment 2, the preview is the vertices, terminations and midsegment defined based on the stroke unit under different masking degrees. The results show that the masking degree is significant.

The vertices and midsegment based on stroke unit definition also have an impact on Chinese character recognition. Different from experiment 1 , the termination based on stroke unit definition also significantly increase the RTs. 
In conclusion, from the above two experiments, we can draw a conclusion that, for the masking degree, whether features were based on line definition or based on the stroke unit as the basic unit, the masking degree of $35 \%$ and $55 \%$ will significantly increase the RTs of the participants. For features type, vertices, midsegment and the termination based on the stroke unit all show significant feature effects, which are consistent with the important features in English word [8] [15] [16]. At the same time, we believe these features also verify the neural recycling hypothesis at the level of Chinese character recognition. The neural recycling hypothesis holds that [17] [18]: 1) Word recognition is derived from object recognition neural circuit recycling [19] [20]. The Area of Visual Word Form Area (VWFA) is adjacent to and partially coincides with the object recognition brain Area, which indirectly proves this [21]. 2) While brain regions may be functionally specialized, neural recycling structures will acquire new features such font invariant and the orthographic rule [20] [22]. So the neural recycling hypothesis is quite different from the theory that reading does not require long-term functional specialization. The key features in research 1 and research 2 prove that word recognition depends on the same features in object recognition. Therefore, we have not evolved a brain region for reading specifically, and the reading system itself is differentiated from the basic visual region [23] [24] [25].

But why is there a significant feature effect on the terminations based on the stroke unit, but not based on the line? This may be mainly due to the way of masking in experiment 2 and experiment 3; only difference of masking terminations between experiment 2 and experiment 3 is that we add hook features in experiment 3. Hook in Chinese characters is a special type of termination, and the hook feature in research 2 shows its importance in research 2.

\section{Limitations}

Now, the experiments presented in this paper demonstrate that vertices, midsegments and terminations are important features in reading and Chinese strokes may play a key role in these features in reading. However, there also have some limitations on this study. First of all, we didn't compare the feature masking stimuli and casual masking stimuli and thus the results of study may result from the pure masking and don't result from feature effect. Second, although delayed segment technique may be similar to real reading, naming task is not real reading process.

\section{Conflicts of Interest}

The authors declare no conflicts of interest regarding the publication of this paper.

\section{References}

[1] Flores d'Arcais, G.B. (1994) Order of Strokes Writing as a Cue for Retrieval in Reading Chinese Characters. European Journal of Cognitive Psychology, 6, 337-355. 
https://doi.org/10.1080/09541449408406519

[2] Yan, G., et al. (2012) Using Stroke Removal to Investigate Chinese Character Identification during Reading: Evidence from Eye Movements. Reading and Writing, 25, 951-979. https://doi.org/10.1007/s11145-011-9295-x

[3] Tseng, S.-C., Chang, L. and Hsiang, W.C.-C. (1965) An Informational Analysis of the Chinese Language: I. The Reconstruction of the Removed Strokes of the Ideograms in Printed Sentence-Texts. Acta Psychologica Sinica, 9, 11-20.

[4] Zhang, J.W., Zhang, H.P. and Zhang, M. (2002) The Effect of the Complexity and Repetition of the Strokes on the Cognition of the Strokes and the Chinese Characters. Acta Psychologica Sinica, 34, 7-11.

[5] Selfridge, O. (1959) Pandemonium: A Paradigm for Learning, Proceedings of Symposium on the Mechanization of Thought Processes. National Physics Laboratory, Teddington.

[6] Fiset, D., et al. (2008) Features for Identification of Uppercase and Lowercase Letters. Psychological Science, 19, 1161-1168. https://doi.org/10.1111/j.1467-9280.2008.02218.x

[7] Lanthier, S.N., et al. (2009) Not All Visual Features Are Created Equal: Early Processing in Letter and Word Recognition. Psychonomic Bulletin \& Review, 16, 67-73. https://doi.org/10.3758/PBR.16.1.67

[8] Rosa, E., Perea, M. and Enneson, P. (2016) The Role of Letter Features in Visual-Word Recognition: Evidence from a Delayed Segment Technique. Acta Psychologica, 169, 133-142. https://doi.org/10.1016/j.actpsy.2016.05.016

[9] Fiset, D., et al. (2009) The Spatio-Temporal Dynamics of Visual Letter Recognition. Cognitive Neuropsychology, 26, 23-35. https://doi.org/10.1080/02643290802421160

[10] Chang, Y., Furber, S. and Welbourne, S. (2012) Modelling Normal and Impaired Letter Recognition: Implications for Understanding Pure Alexic Reading. Neuropsychologia, 50, 2773-2788. https://doi.org/10.1016/j.neuropsychologia.2012.07.031

[11] Lee, H.-W., Rayner, K. and Pollatsek, A. (2002) The Processing of Consonants and Vowels in Reading: Evidence from the Fast Priming Paradigm. Psychonomic Bulletin Review, 9, 766-772. https://doi.org/10.3758/BF03196333

[12] Carreiras, M., et al. (2008) Are Vowels and Consonants Processed Differently? Event-Related Potential Evidence with a Delayed Letter Paradigm. Journal of Cognitive Neuroscience, 21, 275-288. https://doi.org/10.1162/jocn.2008.21023

[13] Lee, H.-W., Rayner, K. and Pollatsek, A. (2001) The Relative Contribution of Consonants and Vowels to Word Identification during Reading. Journal of Memory and Language, 44, 189-205. https://doi.org/10.1006/jmla.2000.2725

[14] Perea, M., Comesaña, M. and Soares, A.P. (2012) Does the Advantage of the Upper Part of Words Occur at the Lexical Level? Memory and Cognition, 40, 1257-1265. https://doi.org/10.3758/s13421-012-0219-Z

[15] Petit, J.-P. and Grainger, J. (2002) Masked Partial Priming of Letter Perception. Visual Cognition, 9, 337-353. https://doi.org/10.1080/13506280042000207

[16] Perea and Manuel (2013) Why Does the APA Recommend the Use of Serif Fonts? Psicothema, 25, 13-17.

[17] Dehaene, S. (2005) Evolution of Human Cortical Circuits for Reading and Arithmetic: The "Neuronal Recycling" Hypothesis. In: Dehaene, S., Duhamel, J.R., Hauser, M. and Rizzolatti, G., Eds., From Monkey Brain to Human Brain, MIT Press, Cambridge, 133-157.

[18] Dehaene, S. and Cohen, L. (2007) Cultural Recycling of Cortical Maps. Neuron, 56, 
384-398. https://doi.org/10.1016/j.neuron.2007.10.004

[19] Cohen, L., et al. (2000) The Visual Word Form Area: Spatial and Temporal Characterization of an Initial Stage of Reading in Normal Subjects and Posterior Split-Brain Patients. Brain, 123, 291-307. https://doi.org/10.1093/brain/123.2.291

[20] Gaillard, R., et al. (2006) Direct Intracranial, FMRI, and Lesion Evidence for the Causal Role of Left Inferotemporal Cortex in Reading. Neuron, 50, 191-204. https://doi.org/10.1016/j.neuron.2006.03.031

[21] Hasson, U., et al. (2002) Eccentricity Bias as an Organizing Principle for Human High-Order Object Areas. Neuron, 34, 479-490. https://doi.org/10.1016/S0896-6273(02)00662-1

[22] Vinckier, F., et al. (2007) Hierarchical Coding of Letter Strings in the Ventral Stream: Dissecting the Inner Organization of the Visual Word-Form System. Neuron, 55, 143-156. https://doi.org/10.1016/j.neuron.2007.05.031

[23] Behrmann, M., Nelson, J. and Sekuler, E. (1998) Visual Complexity in Letter-by-Letter Reading: Pure Alexia Is Not Pure. Neuropsychologia, 36, 1115-1132. https://doi.org/10.1016/S0028-3932(98)00005-0

[24] Price, C.J. and Devlin, J.T. (2003) The Myth of the Visual Word Form Area. Neuroimage, 19, 473-481. https://doi.org/10.1016/S1053-8119(03)00084-3

[25] Cohen, L. and Dehaene, S. (2004) Specialization within the Ventral Stream: The Case for the Visual Word Form Area. Neuroimage, 22, 466-476.

https://doi.org/10.1016/j.neuroimage.2003.12.049 